\title{
Elevated human chorionic gonadotropin HCG serum level in germ cell testicular tumor
}

\section{Opinion}

Testicular cancer is cancer that develops in the testicles, More than $90 \%$ of cancers of the testicle develop is germ cell tumors. These are the cells that make sperm. The 2 main types of germ cell tumors (GCTs) in men are:

a. Seminomas

b. Non-seminomas, which are made up of embryonal carcinoma, yolk sac carcinoma, choriocarcinoma, and/or teratoma. ${ }^{1}$

One of the most important diagnostic methods is laboratory-based tests are potentially useful in screening for early malignancy, aiding cancer diagnosis, determining prognosis, surveillance following curative surgery for cancer, Tumor markers are playing an increasingly important role in cancer detection and management.

In case of testicular cancer diagnosis/case finding, staging, prognosis determination, alpha-fetoprotein (AFP), human chorionic gonadotropin (HCG), and lactate dehydrogenase (LDH) are recommended, they play an important role as serum tumor markers for in the staging and monitoring of germ cell tumors. ${ }^{2}$

\section{But what the relationship between HCG serum levels and germ cell testicular tumors?}

\section{Human chorionic gonadotropin (HCG)}

HCG is a glycoprotein hormone composed of two dissimilar subunits, $\alpha$ and $\beta$, joined noncovalently. It is produced by trophoblast tissue in pregnancy and trophoblast disease and in small amounts by certain poorly differentiated cancers. The $\alpha$-subunit of HCG is similar to that of the pituitary glycoprotein hormones. It is produced principally by the trophoblastic cells of the placenta but may also be produced by nontrophoblastic tissues including normal pituitary and neoplastic cells. ${ }^{3}$ It is normal for non-pregnant women and men to make very small amounts of HCG, with blood levels less than $5 \mathrm{mIU}$ / $\mathrm{mL}$. If HCG is high in non-pregnant women or men, it may indicate cancer. ${ }^{4}$ Where used in malignant causes as tumor marker which is secreted by some cancers including choriocarcinoma, endometrium, and ovarian carcinoma. HCG is elevated in most type of testicular cancer seminomas and non-seminoma germ cell testicular tumors.

It has been found that HCG possesses a role in the angiogenic process in vivo and in vitro by increasing capillary formation and endothelial cell migration, also, hCG-induced neovascularization. HCG may regulate vascular neoformation through vascular endothelial growth factor (VEGF)..$^{5}$

HCG contains five acidic asparagines-linked sugar chains. These five sugar chains are derived by sialylation from three neutral oligosaccharides: two biantennary ( $\mathrm{N}-1$ and $\mathrm{N}-2)$ and one monoantennary (N-3) complex-type oligosaccharides. The malignant transformational change of the sugar moiety of HCG can be explained by an increase of a fucosyltransferas. ${ }^{6}$ Which indicate that N-acetylglucosaminyltransferase IV (GnT-IV) is abnormally expressed in the malignant cells. ${ }^{7}$

\author{
Volume 7 Issue I - 2018
}

\begin{abstract}
Alaa a Elnour
Medical Laboratory Scientist, National Ribat University, Saudi Arabia
\end{abstract}

Correspondence: Alaa a Elnour, Medical Laboratory Scientist, Ibrahim Malik Teaching Hospital National Ribat University, Saudi Arabia,Email alaa.a.elnour@outlook.com

Received: January 29, 2018 | Published: February 06, 2018

There are independent variants of HCG, each produced by different cells with separate biological functions. Multiple molecular variants of HCG include intact HCG, nicked HCG (hCGn), free $\beta$-subunit HCG (hCG $\beta$ ), nicked free $\beta$-subunit HCG (hCG $\beta$ ), free $\alpha$-subunit hCG (hCG $\alpha$ ), and the $\beta$-core fragment hCG (hCG $\beta \mathrm{cf}$ ). In addition, HCG is differentially glycosylated in various tissues, resulting in a range of molecular forms from hypoglycosylated to hyperglycosylated. So-called hyperglycosylated HCG (hCG-h) is probably the best known of these glycosylated variants, ${ }^{8}$ each one of them sharing the common amino acid sequence but each differing in meric structure and carbohydrate side chain structure.

Hcg that produced by trophoblast cells take over corpus luteal progesterone production rom luteinizing hormone (LH) Free b-subunit is the alternatively glycosylated monomeric variant of hCG made by all non-trophoblastic advanced malignancies. Free bsubunit promotes growth and malignancy of advanced cancers, Stimulates implantation by invasion of cytotrophoblast cells as occurs at implantation of pregnancy, blocks apoptosis, growth and malignancy of choriocarcinoma cells. ${ }^{9}$ Therefore, a question, Do all type of variants hCG linked with malignancy?

Unfortunately, studies on hCG-h in testicular cancer are limited, most studies have shown that the most variants of HCG that has related to testicular cancer is hyperglycosylated hCG (hCG-h). Most of the HCG expressed by testicular cancers is hyperglycosylated at diagnosis stage, preoperatively, at relapse, and shortly after treatment. ${ }^{10}$

Hyperglycosylated human chorionic gonadotropin (hCG-H) is a carbohydrate with double-sized oligosaccharide side chains; glycosylation variant of hCG, heterogeneous molecule produced is produced exclusively by stem cytotrophoblast cells, by trophoblastic cells in pregnancy and in gestational trophoblastic diseases including cancer of the trophoblastic cell or choriocarcinoma. It is also produced by other trophoblast sources including testicular germ cell malignancies. Regular HCG is produced only by differentiated syncytiotrophoblast cells. ${ }^{10-12}$ it is also expressed as particular glycoforms by certain malignancies. These glycoforms, which are called "hyperglycosylated" HCG (hCGh), have been reported to contain more complex glycan moieties. ${ }^{13}$

A hyperglycosylated free beta-subunit is produced by a high proportion of all malignancies. This also functions as an autocrine 
by promoting the growth and invasion of the malignancy, primarily hyperglycosylated HCG is produced in early pregnancy and in choriocarcinoma and germ cell testicular malignancies. Only free beta-subunits may be produced in other germ cell malignancies. ${ }^{14}$

Choriocarcinoma cells not only synthesize human chorionic gonadotropin (HCG), but also express $\mathrm{LH} / \mathrm{CG}$ receptors on the cell membrane. $\mathrm{HCG}$ and $\mathrm{LH} / \mathrm{CG}$ receptors may play a role in regulating the biological function of choriocarcinoma cells in an autocrine/ paracrine manner. HCG, through its binding to the $\mathrm{LH} / \mathrm{CG}$ receptor, may augment proliferation and inhibit apoptosis in choriocarcinoma JAr cells, and that the introduction of an antisense gene may be a potential approach to the inhibition of choriocarcinoma cell growth. ${ }^{15}$

Only small proportions of hCG-H are made in the absence of trophoblast invasion ( $<2 \%$ of total HCG), Four model systems have been used for studying the invasive functions of cytotrophoblast cells. All four cytotrophoblast models produce exclusively hCG-H and small amounts of HCG free b-subunit and no detectable regular HCG. Its established functions are autocrine rather than endocrine, modulating trophoblast cell apoptosis, more resembling those of a cytokine than a hormone. ${ }^{16}$

\section{Acknowledgements}

None.

\section{Conflict of interest}

The author declares no conflict of interest.

\section{References}

1. American cancer society Inc.

2. Sturgeon CM, Duffy MJ, Stenman UH, et al. National Academy of Clinical Biochemistry laboratory medicine practice guidelines for use of tumor markers in testicular, prostate, colorectal, breast, and ovarian cancers. Clin Chem. 2008;54(12):e11-e79.

3. Cole LA. Immunoassay of human chorionic gonadotropin, its free subunits, and metabolites. Clin Chem. 1997;43(12):2233-2243.
4. https://www.livestrong.com/article/478036-hcg-levels-cancer/

5. Zygmunt M, Herr F, Keller-Schoenwetter S, et al. Characterization of human chorionic gonadotropin as a novel angiogenic factor. $J$ Clin Endocrinol Metab. 2002;87(11):5290-5296.

6. Kobata A. Structures function, and transformational changes of the sugar chains of glycohormones. J Cell Biochem. 1988;37(1):79-90.

7. Kobata A, Takeuchi M. Structure, pathology and function of the Nlinked sugar chains of human chorionic gonadotropin. Biochim Biophys Acta. 1999;1455(2-3):315-326.

8. Gronowski AM, Grenache DG. Characterization of the hCG Variants Recognized by Different hCG Immunoassays: An Important Step Toward Standardization of hCG Measurements. Clin Chem. 2009;55(8):14471449.

9. Cole LA. Biological functions of hCG and hCG-related molecules. Reprod Biol Endocrinol. 2010;8:102.

10. Cole LA, Khanlian SA, Riley JM, et al. Hyperglycosylated hCG in gestational implantation and in choriocarcinoma and testicular germ cell malignancy tumorigenesis. J Reprod Med. 2006;51(11):919-929.

11. Cole LA. Hyperglycosylated hCG. Placenta. 2007;28(10):977-986.

12. Cole LA, Dai D, Butler SA, et al. Gestational trophoblastic diseases: 1. Pathophysiology of hyperglycosylated hCG. Gynecol Oncol. 2006;102(2):145-150.

13. Valmu L, Alfthan H, Hotakainen K, et al. Site-specific glycan analysis of human chorionic gonadotropin beta-subunit from malignancies and pregnancy by liquid chromatography-electrospray mass spectrometry. Glycobiology. 2006;16(12):1207-1218.

14. Cole LA. Human chorionic gonadotropin tests. Expert Rev Mol Diagn. 2009;9(7):721-747.

15. Hamada AL, Nakabayashi K, Sato A, et al. Transfection of antisense chorionic gonadotropin beta gene into choriocarcinoma cells suppresses the cell proliferation and induces apoptosis. J Clin Endocrinol Metab. 2005;90(8):4873-4879.

16. Cole LA. Hyperglycosylated hCG. Placenta. 2010;31(8):653-664. 